\title{
Por que o orifício em uma chapa se dilata de forma igual à própria chapa?+*
}

\author{
Marcelo Nunes Coelho ${ }^{1}$ \\ André de Oliveira Girão Maia² \\ Instituto Federal de Educação Ciência e Tecnologia do Rio Grande do Norte \\ Mossoró - RN \\ Simony Maia Vieira ${ }^{3}$ \\ Doutoranda em Engenharia Civil - Universidade Federal do Ceará \\ Fortaleza - CE
}

\section{Introdução}

A dilatação térmica é um fenômeno que ocorre em sólidos, líquidos e gases em decorrência de uma variação de temperatura produzida neles. É um tema que faz parte de todos os currículos do ensino médio atualmente, além de ser tema impreterível em cursos superiores de física e engenharias.

Tal fenômeno deve ser levado em consideração em diversas situações enfrentadas por profissionais das áreas de engenharia civil, engenharia mecânica, engenharia elétrica, tendo em vista que todos esses profissionais, vez por outra, tratam de situações que envolvem a variação de temperatura de alguns componentes.

Diversos livros de física oferecem alguma abordagem relacionada ao tema (HALLIDAY; RESNICK; WALKER, 2009; SANT'ANNA et al., 2010; NUSSENZVEIG, 2002; YOUNG; FREEDMAN, 2008, GASPAR, 2011). Em alguns deles, a abordagem é bem superficial (HALLIDAY; RESNICK; WALKER, 2009; SANT'ANNA et al., 2010; GASPAR, 2011); em outros um pouco mais aprofundada (NUSSENZVEIG, 2002; YOUNG; FREEDMAN, 2008). Poucos desses livros, no entanto, respondem as perguntas recorrentes em diversas salas de aula, tais como: "Por que um orifício em uma chapa sólida dilata de forma idêntica à própria chapa?", "Por que o orifício não diminui de tamanho ao aquecermos a chapa?".

Este texto fornecer alternativas para responder a estas perguntas. Sugerimos duas formas: i) justificativa por absurdo, e; ii) matematicamente. Trataremos somente de sólidos com

\footnotetext{
${ }^{+}$Why the hole in a sheet expands equally to their own plate?

* Recebido: agosto de 2015.

Aceito: outubro de 2016.

1 E-mail: marcelonunes06@hotmail.com

2 E-mail: andre.maia@ifrn.edu.br

3 E-mail: simonymv@gmail.com
} 
expansão térmica positiva e isotrópicos (curiosamente, existem materiais cujo coeficiente de dilatação não é o mesmo em todas as direções - por exemplo, o tungstato de alumínio (ACHARY et al., 2002), além daqueles que apresentam comportamento contrário, ou seja, dilatam-se quando resfriados e contraem-se quando aquecidos - por exemplo o tungstato de zircônio (LIND, 2012; ACHARY et al., 2002; EVANS, 1999) ${ }^{4}$.

\section{A dilatação térmica dos sólidos}

Um potencial que descreve com boa aproximação a energia de interação entre partículas de um sólido é dado pela expressão proposta por Lennard-Jones (por isso chamado de potencial de Lennard-Jones):

$$
U(r)=4 E_{p}\left[\left(\frac{a}{r}\right)^{12}-\left(\frac{a}{r}\right)^{6}\right]
$$

Nesta expressão, $E_{p}$ representa a energia potencial de equilíbrio $\left(U\left(r_{\min }\right)=E_{p}\right)-$ a energia que o sistema teria na temperatura de zero absoluto,$- a$ tem dimensões de comprimento e $r$ é a distância instantânea entre as duas partículas.

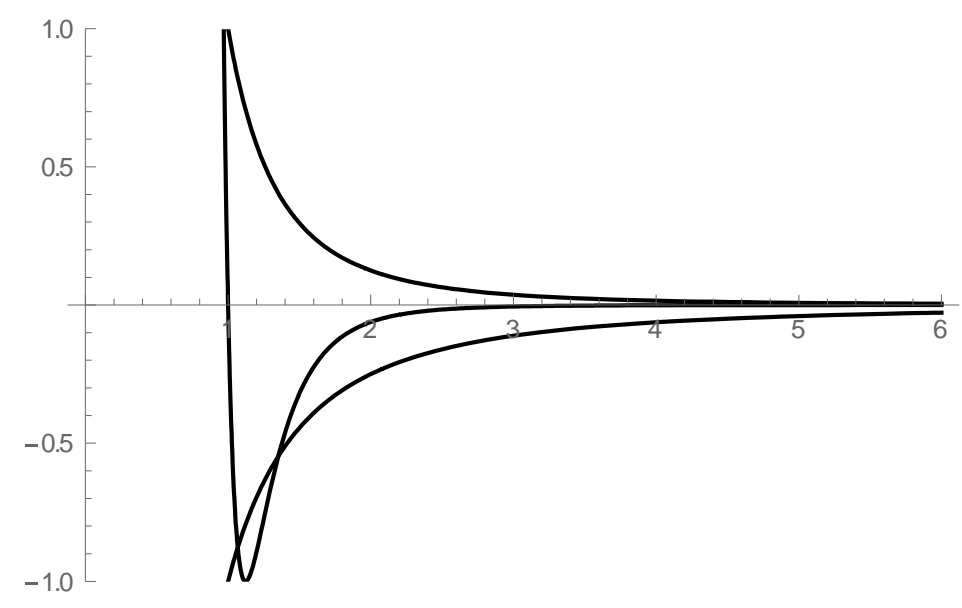

Fig. 1 - Os termos de repulsão (coulombiano) e de atração (Van der Waals) e a combinação dos dois, dando origem ao potencial de Lennard-Jones.

O potencial adquire uma forma assimétrica devido as contribuições combinadas dos efeitos de repulsão (de origem coulombiana) e atração (forças de Van der Waals) (Fig. 1). A observação atenta da forma desse potencial revela que uma elevação de temperatura - o que gera um aumento na energia do sistema -, produz um aumento das distâncias médias entre as posições de equilíbrio das partículas (que passam de $r_{\text {min }}$ - a distância média no zero absoluto

\footnotetext{
${ }^{4}$ Essa classe de materiais tem se mostrado importante na busca por materiais com coeficiente de dilatação nulo, tendo em vista que, ao associar dois materiais - um que dilata positivamente e outro que dilata negativamente - é possível obter um material que não se dilate.
} 
- para $r_{T}$ - a distância média a uma temperatura $T$ qualquer), além do aumento na vibração, que é o responsável pelo aumento de temperatura. Esse acréscimo nas distâncias é o que, ao nível macroscópico, gera a dilatação térmica.

De uma forma geral, dado um objeto com volume inicial $V_{0}$, coeficiente de dilatação volumétrica $\gamma$ (isotrópico, portanto $\gamma=3 \alpha$ ), submetendo-o a uma variação de temperatura $\Delta T$, produz-se uma dilatação volumétrica dada, com boa aproximação, por

$$
\Delta V=V_{0} \cdot \gamma \cdot \Delta T
$$

Esta expressão mostra que um aquecimento $(\Delta T>0)$ gera uma dilatação positiva ao passo que um resfriamento $(\Delta T<0)$ gera uma dilatação negativa (contração) ${ }^{5}$.

\section{O que pensam os alunos, a priori, sobre a expansão de orifícios?}

O problema que iremos analisar, por simplicidade, trata de uma chapa retangular de lados $X$ e $Y$ (chamaremos de lados externos). A placa é constituída de um material isotrópico de coeficiente de dilatação linear $\alpha$. Há, no seu centro, um orifício, também de forma retangular, com lados medindo $x$ e $y$ (chamaremos de lados internos).

Os alunos se convencem facilmente de que os lados externos aumentam com o aumento de temperatura. Resistem, no entanto, a convencerem-se de que os lados internos também dilatam-se. Um argumento que muitos usam para justificar sua dificuldade em aceitar tal fato é o seguinte: imagine que a chapa tenha sido fatiado como na Fig. 2a. Separemos uma dessas fatias (de comprimento $(X-x) / 2$ e largura desprezível) e observemos sua dilatação em separado Fig. 2b. Como essa fatia (muito fina) tem largura desprezível se comparado com o seu comprimento, estamos tratando de um problema de dilatação linear. Assim, será fácil observar a fatia dilatar-se nos dois sentidos. Concluem os alunos, portanto, que se cada fatia da placa dilata-se para dentro e para fora, a placa como um todo aumenta, ao passo que o orifício diminui.

$\mathrm{O}$ argumento parece bom, mas uma análise bem cuidadosa revelará que, apesar da premissa ser aproximadamente verdadeira, a conclusão é errônea.

\section{Por que o buraco se dilata como se fosse feito do mesmo material que a placa onde ele foi feito?}

Como mencionamos anteriormente, forneceremos duas possíveis explicações para o fenômeno. A primeira delas, que será uma demonstração por absurdo, convencerá ao aluno de que o buraco não diminui de tamanho; a segunda, mostrará matematicamente que o buraco dilata exatamente como a placa em que foi feito.

\footnotetext{
5 No caso de podermos desprezar duas das dimensões, teremos um problema de dilatação linear e, no caso de podermos desprezar uma das dimensões, um problema de dilatação superficial.
} 


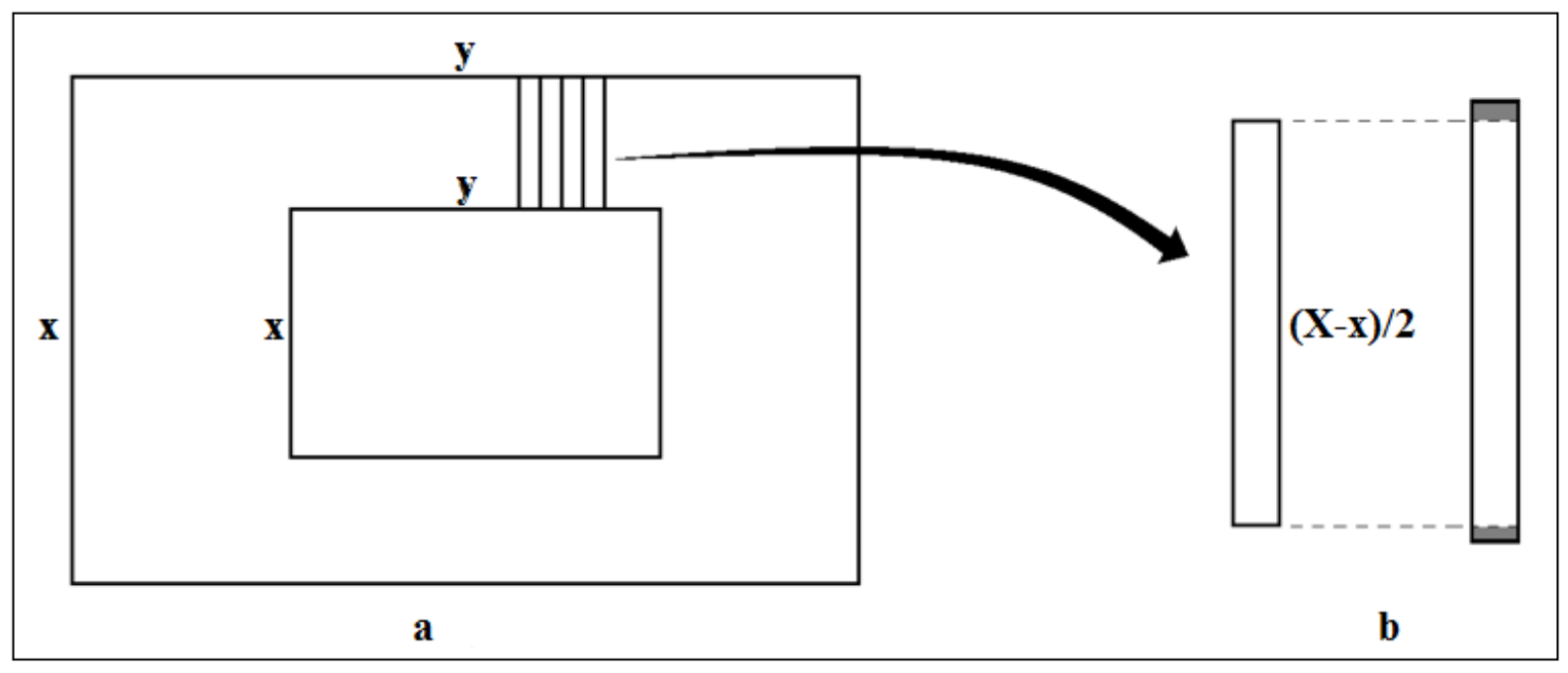

Fig. 2 - a) Uma chapa retangular com um orifício retangular, dividida em fatias de largura desprezível; b) uma dessas fatias em destaque, observando sua dilatação linear.

\section{i) Uma fonte infinita de material}

Queremos mostrar que o furo se dilata. No entanto, vamos mostrar que é impossível ele diminuir. Partiremos, para tanto, da hipótese contrária. Imagine então, que o furo diminui. Seja uma placa sólida da qual extraímos um pequeno quadrado do seu centro. A placa ficará, dessa forma, com um furo também quadrado muito pequeno no seu centro. De acordo com nossa hipótese, ao aquecermos o sistema, a placa como um todo aumenta de tamanho, ao passo que o buraco irá diminuir. Se continuarmos aquecendo, em determinado momento o furo terá fechado. Podemos, novamente, extrair outro quadrado muito pequeno do seu centro, produzindo um novo furo na placa. Após determinada variação positiva de temperatura, o furo terá fechado. Observe que, apesar de estarmos extraindo material da placa, a mesma como um todo, está aumentando de tamanho. Dessa forma, teríamos uma fonte infinita de material. Como essa fonte infinita de material é impossível, conclui-se que nossa hipótese (a de que o furo diminui) é falsa.

\section{ii) A placa que some}

Se acreditamos que o buraco diminui quando a placa se dilata, é razoável crer que ele aumenta quando a placa esfria. No entanto, se isso for possível, estando a placa diminuindo com o resfriamento e o buraco aumentando, chegará um momento que o buraco terá o tamanho da placa. A placa sumiu! Isso também não faz sentido. Assim sendo, temos mais um motivo para acreditar que nossa hipótese inicial é falsa. 


\section{iii) $O$ furo realmente se dilata da mesma forma que a placa em que ele foi feito}

Para simplificar, vamos supor uma placa retangular de lados medindo $X_{0}$ e $Y_{0}$ inicialmente, com um furo também retangular de lados medindo $x_{0}$ e $y_{0}$ inicialmente. A Fig. 3 ilustra a situação.

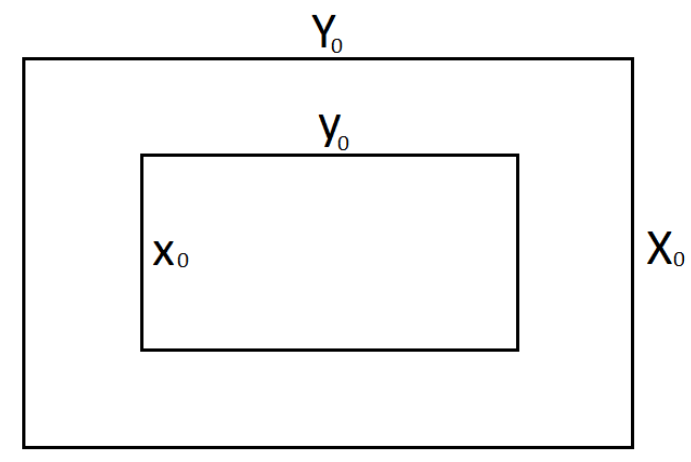

Fig. 3 - Uma placa retangular de lados $X_{0}$ e $Y_{0}$ com um orifício retangular de lados $x_{0}$ e $y_{0}$.

Após aquecermos a placa, cada lado externo estará medindo

$$
X=X_{0}(1+\alpha \Delta T) \text { e } Y=Y_{0}(1+\alpha \Delta T)
$$

A área total delimitada por essa nova superfície, é dada por

$$
X Y=X_{0} Y_{0}(1+2 \alpha \Delta T)
$$

Na qual usamos a aproximação da série binomial para $(1+\alpha \Delta T)^{2}$, tendo em vista que $\alpha \Delta T$ é muito menor que 1 . Isso pode ser reescrito como

$$
X Y=X_{0} Y_{0}(1+\beta \Delta T)
$$

sendo $\beta=2 \alpha$.

Sejam $A_{1}$ e $A_{2}$ as áreas das superfícies delimitadas na Fig. 4. É fácil ver que, após a dilatação

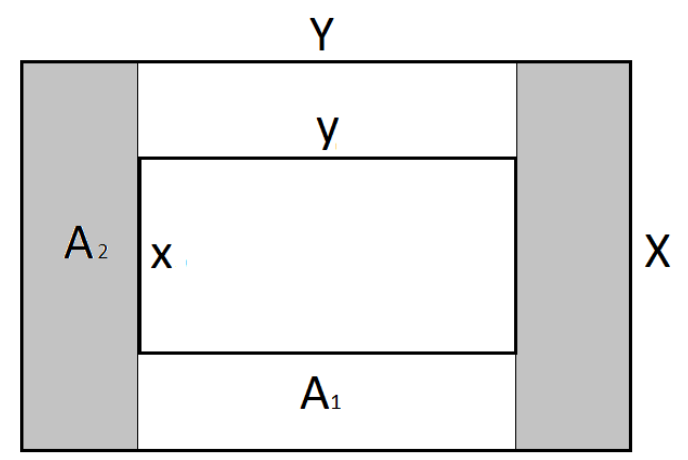

Fig. 4 - A mesma placa da figura anterior depois de dilatada. Destacam-se as subáreas $A 1$ e $A 2$. 


$$
\begin{aligned}
& A_{1}=y_{0} \frac{X_{0}-x_{0}}{2}(1+\beta \Delta T) \\
& A_{2}=X_{0} \frac{Y_{0}-y_{0}}{2}(1+\beta \Delta T)
\end{aligned}
$$

A área do buraco $A_{b}$ é dada por

$$
A_{b}=X_{0} Y_{0}(1+\beta \Delta T)-Y_{0}\left(X_{0}-x_{0}\right)(1+\beta \Delta T)-x_{0}\left(Y_{0}-y_{0}\right)(1+\beta \Delta T)
$$

Rearranjando os termos, obtemos

$$
A_{b}=x_{0} y_{0}(1+\beta \Delta T)
$$

Que é exatamente a área final de uma placa retangular de lados medindo inicialmente $x_{0}$ e $y_{0}$ com coeficiente de dilatação superficial $\beta$.

\section{Conclusão}

Conseguimos mostrar que um orifício em uma placa dilata-se da mesma forma que a placa. A demonstração que poderia ser feita em um único passo (o matemático), foi levada a cabo por meio de duas etapas: na primeira, provamos por absurdo que o furo não pode diminuir e, por fim, mostramos matematicamente que o furo dilata-se da mesma forma que o material da placa em que ele foi feito.

Nota-se que, de fato, as hipóteses que os alunos usam para justificar suas impressões de que o furo deve diminuir, são válidas. Contudo, as conclusões são erradas porque eles esquecem de levar em conta algo mais. Apesar de cada fatia da placa dilatar-se (para dentro e para fora) como se dilata uma barra, o fato de o material ter que se dilatar como um todo gera uma competição entre essas duas dilatações, produzindo um efeito líquido de dilatação do orifício.

\section{Referências Bibliográficas}

HALLIDAY, D.; RESNICK, R.; WALKER, J. Fundamentos de Física. Rio de Janeiro: LTC, 2009. 188 p.

SANT'ANNA, B. et al. Conexões com a Física. São Paulo: Moderna, 2010. 59 p.

NUSSEnZVEIG, H. M. Curso de Física Básica. São Paulo: Edgard Blucher, 2002. 163 p.

YOUNG, H. D.; FREEDMAN, R. A. Física II. São Paulo: Addison Wesley, 2008. 188 p.

GASPAR, A. Compreendendo a Física. São Paulo: Ática, 2011. 272 p.

LIND, C. Two decades of negative thermal expansion Research: where do we stand? Materials, v. 5, p. 1125-1154, jun. 2012. 
ACHARY, S. N. et al. Preparation, thermal expansion, high pressure and high temperature behavior of $\mathrm{Al}_{2}\left(\mathrm{WO}_{4}\right)_{3}$. Journal of Materials Science, v. 37, p. 2501-2509, fev. 2002.

EVANS, J. S. O. Negative thermal expansion materials. Journal of Chemical Society, Dalton Transactions, v. 19, p. 3317-3326, jul. 1999. 